\title{
Surface magnetic properties and domains observation in as-quenched and annealed FeNbB ribbons
}

\author{
O. Životskýa ${ }^{\text {a,* }}$, K. Postava ${ }^{\mathrm{a}}$, L. Kraus ${ }^{\mathrm{b}}$, K. Hrabovskáa ${ }^{\mathrm{a}}$, A. Hendrych ${ }^{\mathrm{a}}$, J. Pištora ${ }^{\mathrm{a}}$ \\ ${ }^{\mathrm{a}}$ Department of Physics, Technical University of Ostrava, 17.listopadu 15, 70833 Ostrava-Poruba, Czech Republic \\ ${ }^{\mathrm{b}}$ Institute of Physics, Academy of Sciences of the Czech Republic, Na Slovance 2, 18221 Prague 8, Czech Republic
}

\section{A R T I C L E I N F O}

\section{PACS:}

75.60. Ch;

75.30.Gw;

75.50.Tt;

75.60. Nt

Keywords:

Magneto-optic

Kerr effect;

Magnetic

domains; Magnetic

anisotropy;

Amorphous and

nanocrystalline

ribbons

\begin{abstract}
A B S T R A C T
The longitudinal magneto-optical Kerr effect (MOKE) combined with the magneto-optical microscopy is used to investigate the surface magnetic anisotropy and the domain behavior in as-quenched (AQ) and strain-annealed (SA) FeNbB ribbons. X-ray diffraction, conversion electron Mössbauer spectroscopy (CEMS), and scanning electron microscopy (SEM) confirmed the existence of approximately $1.3 \mu \mathrm{m}$ thick magnetically harder layer on the ribbon air side, consisting of crystallites embedded in an amorphous matrix. The underneath bulk phase is amorphous and magnetically soft. Residual anisotropies and the domain patterns (wide in-plane domains with $180^{\circ}$ walls and narrow fingerprint domains) corresponding to amorphous phase are mainly caused by the internal stresses originating from the rapid quenching process. In the surface crystalline phase they are gradually overlapped by strip domains with magnetization directions almost perpendicular to the ribbon axis. The anisotropy changes caused by additional annealing and straining of the samples are shown and discussed in details.
\end{abstract}

(C) 2009 Elsevier B.V. All rights reserved.

\section{Introduction}

Rapidly quenched Fe-based ribbons show outstanding physical, chemical, and structural properties, which makes them very attractive for various applications as soft magnetic materials [1]. It was shown that the magnetization behavior of such materials strongly depends on their domain structure, which reflects the presence of magnetic anisotropy induced by internal stresses originating during manufacturing process [2]. Stress relaxation and controlled induced anisotropy can be produced by annealing in applied magnetic field [3]. Examples of domain patterns in FeBSi-based and CoFeBSi-based amorphous ribbons can be found in Refs. $[2,4,5]$. There is, however, also a need for detail anisotropy and domain characterization in other Fe-based nanocrystalline materials like the NANOPERM [Fe-M-B (M=Zr, Hf, $\mathrm{Nb})$ ] and the FINEMET (Fe-Si-B-Nb-Cu) type alloys. Some investigations devoted to the relation between the surface nanocrystallization and the magnetic domain structures have already been presented in FINEMET alloys [6,7].

Recently, the surface magnetic behavior of the NANOPERM-type Fe80.5 $\mathrm{Nb}_{6.9} \mathrm{~B}_{12.6}$ ribbons has been investigated [8] using various non-destructive methods. X-ray diffraction, conversion electron Mössbauer spectroscopy (CEMS), and magneto-optical Kerr effect (MOKE) confirmed the partial surface crystallization on both ribbon sides, while the bulk is amorphous. The thickness of thin crystalline layer on the wheel side (the side in direct contact with quenching wheel) was estimated to be only few nanometers.

\footnotetext{
* Corresponding author. Tel: +420597323100 ; fax: +42059732 Email address: ondrej.zivotsky@post.cz (O. Životský).
}

In this paper the surface magnetic properties and the domain structures on the shiny side (the side in contact with the air during preparation process) of FeNbB ribbons are studied using MOKE. The investigation of the shiny side is motivated by the fact that observed crystalline phase penetrates deeper than that on the wheel side and is exchange coupled to the soft amorphous bulk [9], what is of great importance especially in magnetic sensors and recording. Moreover, the shiny surface is smooth enough to allow the domain observations without additional surface treatment.

\section{Experimental}

Fe $80.5 \mathrm{Nb}_{6.9} \mathrm{~B}_{12.6}$ ribbons, $10 \mathrm{~mm}$ wide and $28 \mu \mathrm{m}$ thick, were prepared by the planar flow casting method. Two different samples were investigated: the as-quenched sample (AQ) and the strain-annealed one (SA). To reduce the influence of undesirable shape anisotropy the AQ sample, in the shape of circular disc with diameter of $9 \mathrm{~mm}$, was spark-cut from the as-quenched ribbon. The SA sample was taken from the ribbon coiled, with the shiny side in, around the silica tube with diameter of $13 \mathrm{~mm}$ and stress relieved in air for 30 minutes at $380^{\circ} \mathrm{C}$. After annealing the SA sample preserves the toroidal shape. For the measurements it was unwound and fixed on a planar sample holder with the shiny side up. By this procedure inhomogeneous stress is produced in the ribbon, which changes from the tensile stress (along the ribbon axis) on the shiny side to the compressive one on the wheel side.

Our experimental setups combine the hysteresis loop and the domain observation techniques, both based on the longitudinal MOKE. Differential intensity method consisting of the red semiconductor laser 
a

longitudinal configuration

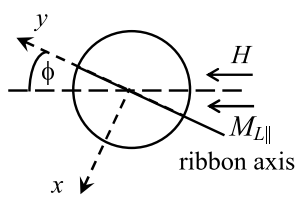

transversal configuration

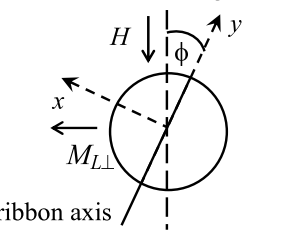

Fig. 1. MOKE configurations used for the in-plane magnetization measurements. $H$ denotes the applied magnetic field, which makes the angle $\phi$ with the ribbon axis. Subplots (a) and (b) correspond to magnetization component $M_{L \|}$ and $M_{L \perp}$ parallel and perpendicular to $H$, respectively.

at the wavelength of $670 \mathrm{~nm}$, the polarizer, the Wollaston prism, and two photodiodes are used for surface hysteresis loops measurements. Approximately circular laser spot about $0.5 \mathrm{~mm}$ in diameter was focused on the center of the ribbon. Using this method we measure both in-plane magnetization components that are distinguished by the rotation of the sample and magnetic field by $90^{\circ}$ [10], as shown in Figure 1. Specially designed sample holder ensures that the position of the laser spot does not change during the sample rotation.

Magnetic domain observations were carried out with the Zeiss optical microscope adapted for the Kerr magneto-optical microscopy. White light from the arc xenon lamp goes through the polarizer and polarization objective. After reflection from the sample it is transmitted through the analyzer almost crossed with the polarizer. A digitized video image of the magnetically saturated state obtained by the high resolution CCD camera is subtracted from the pictures containing magnetic contrast [2]. The resulting difference image is then improved by averaging.

\section{Results and discussions}

\subsection{As-quenched ribbon}

Figure 2 shows the proposed model of shiny side of AQ ribbon consisting of two-layered system. The presence of crystalline layer on the ribbon surface was confirmed by the X-ray diffraction and the CEMS, which detected about $5 \%$ of crystalline $\alpha$-Fe phase in region of the thickness about 200-300 $\mathrm{nm}$ [8]. Longitudinal hysteresis loop of crystalline layer, shown in Fig. 2, was measured for $s$-polarized incident light at incident angle of $60^{\circ}$ in configuration $\phi=0^{\circ}$. Because the loop shape detects the presence of one phase on the surface with the coercive field about 40 Oe it seems that the "effective crystalline layer" extends much deeper than the penetration depth of inspected light $(30 \mathrm{~nm})$. Notation "effective crystalline layer" means that it consists of crystallites more or less randomly embedded in the amorphous phase.

Electrochemical surface polishing was used to remove the crystallites from the surface. Longitudinal MOKE loop of detected bulk phase (see Fig. 2) differed in the shape from the previous one and its coercive field decreased to 10 Oe. By measuring the sample weight before and after polishing we found that about $4.6 \%$ of ribbon thickness had been removed. Based on these results the thickness of effective crystalline layer was estimated to be about $1.3 \mu \mathrm{m}$. This fact was supported also by the metallographic observations. Cross-section image from the scanning electron microscope (SEM) showed a sharp interface between both phases and the thickness of effective crystalline phase of about $1.5 \mu \mathrm{m}$.

Figure 3 shows the zero-field magnetic domain patterns observed
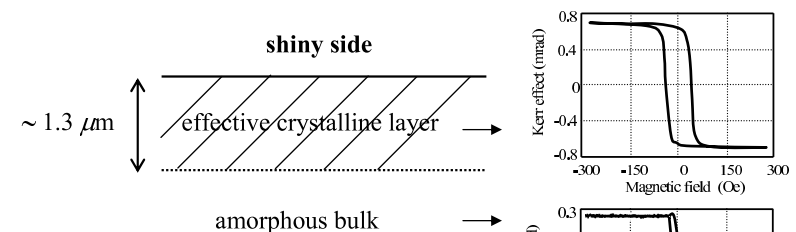

amorphous bulk

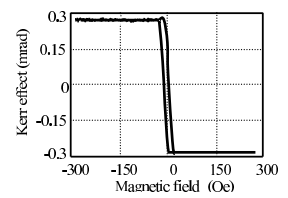

Fig. 2. Model describing the near-surface region from the shiny side of $A Q$ FeNbB ribbon. Longitudinal MOKE loops $\left(\phi=0^{\circ}\right)$ of crystalline and bulk phases are shown at right subplots.

on the polished ribbon surface (bulk phase). In MO microscopy the light emitted from the lamp is focused on the plane of the aperture diaphragm that determines the angle of incidence and plays the key role for adjusting the MOKE configuration [11]. In Fig. 3 we chose the off-centered diaphragm (see upper subplot) which leads to the oblique angle of incidence and the sensitivity to the magnetization component $M_{L \perp}$ parallel to the ribbon axis. Because the ribbon is saturated by external magnetic field $H$ with the direction perpendicular to the $M_{L \perp}$, this case corresponds to the transversal configuration from Fig. 1b, when $\phi=90^{\circ}$. Both domain types, wide curved domains (left side of Fig. 3a) and narrow fingerprint (maze) domains, are caused by the internal stresses originating from the quenching process and are closely related to the magnetostriction constant $\lambda_{s}=10.8 \times 10^{-6}$ measured by the transverse susceptibility method [12]. Random nature of mentioned stresses causes that one or both domain patterns are visible at different places (Figs. 3a,b). The domains with $180^{\circ}$-walls observed in Fig. 3a, that reach the maximum width up to $100 \mu \mathrm{m}$, are the consequence of dominating tensile stress in the ribbon bulk and follow a local in-plane easy axis, which varies over the ribbon surface. On the contrary, the fingerprint domains with the widths up to $5 \mu \mathrm{m}$ are caused mainly by the planar compressive stress. It means that they represent the surface closure domains for the internal domains with preferred magnetization direction perpendicular to the surface. Moreover, the branching phenomenon of maze structures related to the strong stress level was observed at specific regions of the sample, see subplot 3b. Note that similar domain patterns were reported for AQ Metglass ribbons with positive magnetostriction $[2,4,5]$.

Domain structures from two different regions on the non-polished surface (effective crystalline phase) are shown in Fig. 4. The same
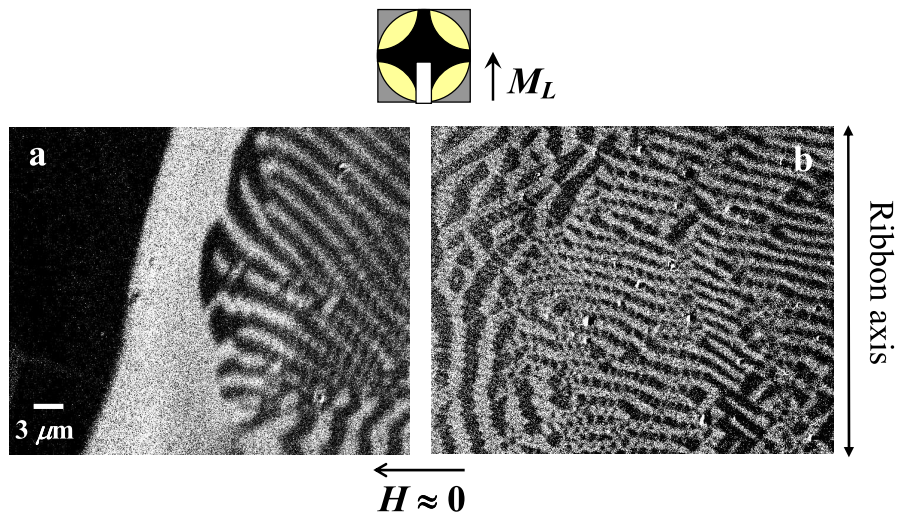

Fig. 3. The magnetic domain patterns observed at polished FeNbB ribbon in two different places. 


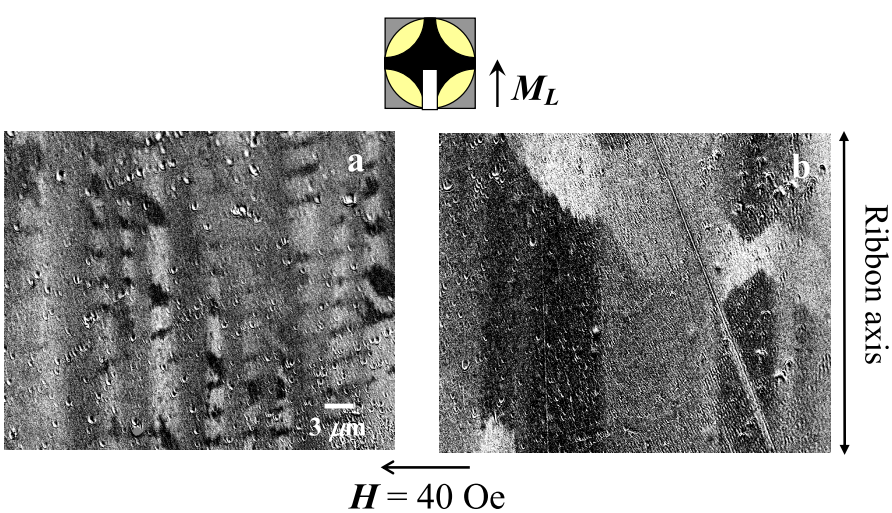

Fig. 4. Detail of magnetic domain in the effective crystalline phase of AQ FeNbB ribbon. Two types of stress-dominated (a-fingerprint), (b-wide curved) domain patterns, observed by focusing the light onto two different points on the ribbon surface, are overlapped with grey strip domains.

magneto-optical configuration as in Fig. 3 is used. After saturating the sample and taking the reference picture, the applied field $H$ is steaply decreased. For $H \approx 0$, the original black and white domains (maze or wide curved ones) known from the bulk phase can be seen. Changing the polarity of $H$ and slightly increasing its magnitude in the opposite direction leads to the patterns, where the original black and white domains become overlapped with grey strip domains, the directions of which are almost perpendicular to the ribbon axis. We assume that the origin of these strip domains may be attributed to the $\alpha$-Fe grains situated near the surface, while the stress patterns with magnetization directions predominantly along the ribbon axis, observed at $H \approx 0$, come from the amorphous bulk phase underneath. These results confirm that magnetic properties of the as-quenched FeNbB ribbon change with the depth from the sample surface.

\subsection{Strain-annealed ribbon}

After strain-annealing the CEMS showed an increase of $\alpha$-Fe phase from $5 \%$ to $13 \%$. Unfortunately, the ribbons are too brittle to be polished, so it is not possible to estimate, how the profile of the crystalline phase distribution has changed. Figure 5 illustrates the magnetic properties of the crystalline phase on the surface of SA sample. As a consequence of tensile stress on the shiny side of unwound SA sample and the positive magnetostriction constant of amorphous phase magnetoelastic anisotropy with the easy direction along the ribbon axis is induced in the amorphous matrix of the effective crystalline layer as well as in the amorphous bulk underneath. Due to the exchange coupling of $\alpha$-Fe grains with the amorphous matrix [11] their local magnetizations also tend to be oriented along the ribbon axis. This is clearly confirmed by the MOKE hysteresis loop measurements. It can be seen that the in-plane easy axis on the shiny side corresponds to the ribbon axis. Loops shown in Figs. 5a,b are normalized to the MOKE signal $1.5 \mathrm{mrad}$. The coercive field of SA sample decreased to 25 Oe (in comparison to 40 Oe of AQ sample). Also the zero-field domain pattern which is observed after strain-annealing (Fig. 5c) is quite different from those observed on AQ samples (Figs. 3 and 4). The fingerprint patterns completely disappeared and the wide strip domains, typical for strong in-plane uniaxial anisotropy, are now oriented parallel to the ribbon axis.
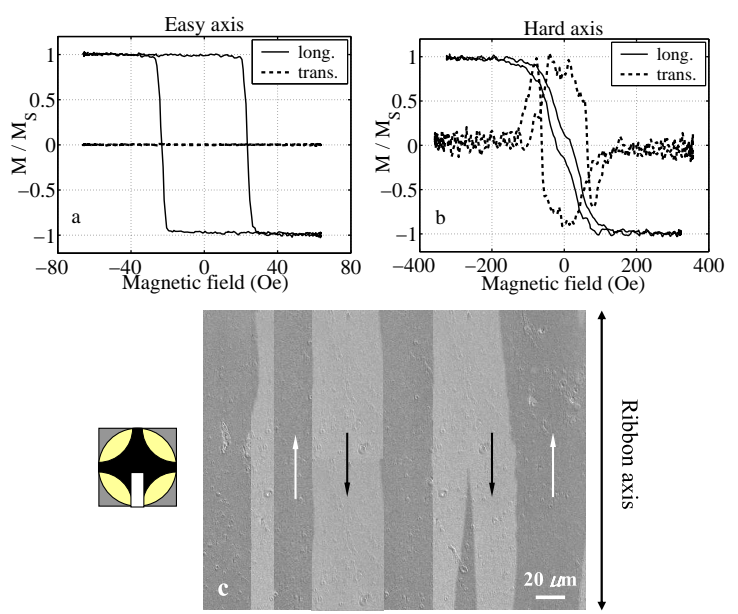

Fig. 5. (a,b) Measured in-plane easy- and near-hard-axis hysteresis loops of annealed FeNbB ribbons in longitudinal and transversal directions. Magnetic field is applied parallel $\left(\phi=0^{\circ}\right)$ and perpendicular $\left(\phi=90^{\circ}\right)$ to the ribbon axis. (c) Corresponding zero-field domain patterns.

\section{Conclusions}

The surface magnetic properties and the domain observations in as-quenched and strain-annealed $\mathrm{Fe}_{80.5} \mathrm{Nb}_{6.9} \mathrm{~B}_{12.6}$ ribbons are studied using the MOKE. As we have shown, the combination of the magnetooptical hysteresis loops measurements with the magneto-optical Kerr microscopy seems to be suitable tool for investigations of surface magnetic anisotropy. While in the bulk phase the internal stresses are the main source of anisotropy, the origin of strip domains overlayed the stress patterns may be related to the the presence of crystallites in near-surface region of the ribbon. Straining the ribbon after the relief annealing induces ordered domain structure and magnetic anisotropy with the in-plane easy axis parallel to the applied tensile stress on the shiny side of the ribbon.

\section{Acknowledgments}

This work has been partially supported by the grants KAN (400100653, MSM6198910016), by the Czech Grant Agency (202/06/0531, 102/08/0743), and by the Czech Academy of Sciences (AVOZ 10100520).

\section{References}

[1] A. Inoue, A. Makino, T. Bitoh, Soft Magnetic Bulk Glassy and Bulk Nanocrystalline Alloys, in: Handbook of Magnetism and Advanced Magnetic Materials, John Wiley \& Sons, 2007.

[2] A. Hubert, R. Schäfer, Magnetic Domains, Springer, New York, 2000.

[3] O.V. Nielsen, H.J.V. Nielsen, Solid State Comm. 35 (1980) 281.

[4] Y. Birol, Turk. J. Phys. 22 (1998) 481.

[5] R. Schäfer, J. Magn. Magn. Mater. 215-216 (2000) 652.

[6] R. Schäfer, A. Hubert, G. Herzer, J. Appl. Phys. 69 (1991) 5325.

[7] S. Flohrer, R. Schäfer, C. Polak, G. Herzer, Acta Mat. 53 (2005) 2937.

[8] O. Životský et al., J. Magn. Magn. Mater. 320 (2008) 1535.

[9] L. Kraus, O. Životský, K. Postava, P. Švec, D. Janičkovič, IEEE Trans. Magn. 44 (2008) 3875

[10] C. Daboo, J.A.C. Bland, R.J. Hicken, A.J.R. Ives, M.J. Baird, and M.J. Walker, Phys. Rev. B 47 (1993) 11852.

[11] R. Schäfer, Investigation of Domains and Dynamics of Domain Walls by the Magneto-optical Kerr-effect, in: Handbook of Magnetism and Advanced Magnetic Materials, John Willey \& Sons, 2007.

[12] L. Kraus, J. Phys. E: Sci. Instrum. 22 (1989) 943. 